\title{
GENERALIZATIONS OF ROSENTHAL'S INEQUALITIES
}

\author{
V.V. Petrov \\ Sankt-Petersburg University, Faculty of Mathematics and Mechanics \\ Stary Peterhof, 198904 St.-Petersburg, Russia
}

1. We shall prove some generalizations of two well-known inequalities for moments of sums of independent random variables obtained by Rosenthal [5], [6]. Instead of the classical power moments we consider moments belonging to a more general class. Another generalization is connected with one-sided moments. We prove some inequalities for generalized moments of this type for the maximum of partial sums of independent random variables.

2. Let $X_{1}, X_{2}, \ldots, X_{n}$ be independent random variables, $S_{k}=\sum_{i=1}^{k} X_{i}$. Let $G_{0}$ be the set of non-negative even functions $g(x), x \in \mathbf{R}$, non-decreasing on the positive half-axis and satisfying $g(0)=0$.

Theorem 1. Suppose

$$
\mathbf{E} X_{k}=0, \quad k=1, \ldots, n,
$$

and

$$
0<B_{n}<\infty
$$

where

$$
B_{n}=\sum_{k=1}^{n} \mathbf{E} X_{k}^{2}
$$

If

$$
\mathbf{E} g\left(X_{k}\right)<\infty, \quad k=1, \ldots, n,
$$

for some $g \in G_{0}$, then

$$
\mathbf{E} g\left(\max _{1 \leq k \leq n} S_{k}\right) \leq \sum_{k=1}^{n} \mathbf{E} g\left(r X_{k}\right)+2 e^{r} \int_{0}^{\infty}\left(1+\frac{x^{2}}{r B_{n}}\right)^{-r} d g(x)
$$

for every $r>0$. 
Remark. For $g(x)=|x|^{p}, x \in \mathbf{R}, p \geq 2$, we obtain

$$
\mathbf{E}\left|\max _{1 \leq k \leq n} S_{k}\right|^{p} \leq r^{p} M_{p, n}+2 p e^{r} r^{p / 2} B\left(\frac{p}{2}, r-\frac{p}{2}\right) B_{n}^{p / 2}
$$

for every $p \geq 2$ and $r>\frac{1}{2} p$, where

$$
M_{p, n}=\sum_{k=1}^{n} \mathbf{E}\left|X_{k}\right|^{p}
$$

and $B(x, y)$ is the Beta-function.

Let $X$ be a random variable with the distribution function $F(x), x \in \mathbf{R}$. In what follows we use the notation

$$
\mathbf{E}^{+} g(X)=\int_{0}^{\infty} g(x) d F(x)
$$

Theorem 2. Suppose

$$
\mathbf{E}^{+} g\left(X_{k}\right)<\infty, \quad k=1, \ldots, n
$$

for some $g \in G_{0}$. If the conditions (1) and (2) are satisfied, then

$$
\mathbf{E}^{+} g\left(\max _{1 \leq k \leq n} S_{k}\right) \leq \sum_{k=1}^{n} \mathbf{E}^{+} g\left(r X_{k}\right)+e^{r} \int_{0}^{\infty}\left(1+\frac{x^{2}}{r B_{n}}\right)^{-r} d g(x)
$$

for every $r>0$.

Theorem 3. Suppose

$$
0<D_{n}<\infty
$$

where

$$
D_{n}=\sum_{k=1}^{n} \mathbf{E}\left|X_{k}\right|
$$

If the condition (4) is satisfied for some $g \in G_{0}$, then

$$
\mathbf{E} g\left(\max _{1 \leq k \leq n} S_{k}\right) \leq \sum_{k=1}^{n} \mathbf{E} g\left(r X_{k}\right)+2 e^{r} \int_{0}^{\infty}\left(1+\frac{x}{D_{n}}\right)^{-r} d g(x)
$$

for every $r>0$. 
Remark. For $g(x)=|x|^{p}, x \in \mathbf{R}, p>1$, we get

$$
\mathbf{E}\left|\max _{1 \leq k \leq n} S_{k}\right|^{p} \leq r^{p} M_{p, n}+2 p e^{r} B(p, r-p) D_{n}^{p}
$$

for every $r>p>1$, where $B(x, y)$ is the Beta-function and $M_{p, n}$ is defined by (7).

Theorem 4. If the conditions (10) and (8) are satisfied for some $g \in G_{0}$, then

$$
\mathbf{E}^{+} g\left(\max _{1 \leq k \leq n} S_{k}\right) \leq \sum_{k=1}^{n} \mathbf{E}^{+} g\left(r X_{k}\right)+e^{r} \int_{0}^{\infty}\left(1+\frac{x}{D_{n}}\right)^{-r} d g(x)
$$

for every $r>0$.

\section{Proof of Theorems 1 and 2.}

Lemma 1. Let $y_{1}, \ldots, y_{n}$ be positive numbers, $y=\max \left\{y_{1}, \ldots, y_{n}\right\}$. If the condition (2) holds, then

$$
\mathbf{P}\left(\max _{1 \leq k \leq n} S_{k} \geq x\right) \leq \sum_{k=1}^{n} \mathbf{P}\left(X_{k} \geq y_{k}\right)+\exp \left\{\frac{x}{y}-\frac{x}{y} \log \left(1+\frac{x y}{B_{n}}\right)\right\}
$$

and

$$
\mathbf{P}\left(\left|\max _{1 \leq k \leq n} S_{k}\right| \geq x\right) \leq \sum_{k=1}^{n} \mathbf{P}\left(\left|X_{k}\right| \geq y_{k}\right)+2 \exp \left\{\frac{x}{y}-\frac{x}{y} \log \left(1+\frac{x y}{B_{n}}\right)\right\}
$$

for every $x>0$.

Lemma 1 follows from inequalities of Fuk and Nagaev [2] and a result of Borovkov [1] (see also Lemma 13, inequality (5.5) and Supplement 16 (Section 6) in Chapter 3 of [4]). then

Lemma 2. If $X$ is a random variable and $\mathbf{E}^{+} g(X)<\infty$ for some $g \in G_{0}$,

$$
\mathbf{E}^{+} g(X)=\int_{0}^{\infty} \mathbf{P}(X \geq x) d g(x) .
$$

If $\mathbf{E} g(X)<\infty$ for some $g \in G_{0}$, then

$$
\mathbf{E} g(X)=\int_{0}^{\infty} \mathbf{P}(|X| \geq x) d g(x) .
$$

It is easy to prove this lemma by integrating by parts the expressions appearing on the right-hand sides of the last two equalities. We take into account also 
the relations $g(0)=0$ and $\lim _{x \rightarrow+\infty} g(x) P(X \geq x)=0$, which follows from the inequality

$$
g(x) \mathbf{P}(X \geq x) \leq \int_{x}^{\infty} g(y) d F(y), \quad x>0,
$$

where $F(y), y \in \mathbf{R}$, stands for the distribution function of $X$.

Let $x>0$ and $r>0$. To prove Theorem 2 we put in Lemma $1 y_{k}=y=x / r$, $k=1, \ldots, n$. We then have

$$
\mathbf{P}\left(\max _{1 \leq k \leq n} S_{k} \geq x\right) \leq \sum_{k=1}^{n} \mathbf{P}\left(r X_{k} \geq x\right)+\exp \left\{r-r \log \left(1+\frac{x^{2}}{r B_{n}}\right)\right\}
$$

and

$$
\int_{0}^{\infty} \mathbf{P}\left(\max _{1 \leq k \leq n} S_{k} \geq x\right) d g(x) \leq I_{1}+I_{2}
$$

where

$$
I_{1}=\sum_{k=1}^{n} \int_{0}^{\infty} \mathbf{P}\left(r X_{k} \geq x\right) d g(x), \quad I_{2}=e^{r} \int_{0}^{\infty}\left(1+\frac{x^{2}}{r B_{n}}\right)^{-r} d g(x) .
$$

Applying Lemma 2 we get

$$
\mathbf{E}^{+} g\left(\max _{1 \leq k \leq n} S_{k}\right) \leq \sum_{k=1}^{n} \mathbf{E}^{+} g\left(r X_{k}\right)+I_{2}
$$

finishing the proof of Theorem 2 .

Theorem 1 can be proved using the other inequalities in Lemma 1 and Lemma 2.

4. The proofs of Theorems 3 and 4 are similar to the proofs of Theorems 1 and 2. Instead of Lemma 1 it is possible to apply the consequences of more general probabilistic inequalities stated in [2], [3] and [1].

5. Lemma 1 remains true if we replace $\max _{1 \leq k \leq n} S_{k}$ by $S_{n}$. Therefore under the conditions of Theorem 1 the same upper bound for $\mathbf{E} g\left(S_{n}\right)$ holds as the one given in (5). In particular, we have

$$
\mathbf{E}\left|S_{n}\right|^{p} \leq C(p)\left(M_{p, n}+B_{n}^{p / 2}\right), \quad p \geq 2
$$

and, taking into account (6),

$$
\mathbf{E}\left|\max _{1 \leq k \leq n} S_{k}\right|^{p} \leq C(p)\left(M_{p, n}+B_{n}^{p / 2}\right), \quad p \geq 2 .
$$


Here $C(p)$ is a positive constant depending only on $p$.

Inequality (15) is due to Rosenthal [5], [6]. Of course Theorems 2, 3 and 4 remain true also if we replace $\max _{1 \leq k \leq n} S_{k}$ by $S_{n}$. In particular, under the conditions of Theorem 3 we have

$$
\mathbf{E}\left|S_{n}\right|^{p} \leq C(p)\left(M_{p, n}+D_{n}^{p}\right), \quad p>1,
$$

and, as a consequence of (13),

$$
\mathbf{E}\left|\max _{1 \leq k \leq n} S_{k}\right|^{p} \leq C(p)\left(M_{p, n}+D_{n}^{p}\right), \quad p>1 .
$$

Inequality (17) was proved by Rosenthal [5], [6].

\section{References}

[1] Borovkov, A.A.: Notes on inequalities for sums of independent variables. - Theory Probab. Appl. 17, 1972, 556-557 (English translation of Teor. Veroyatnost. i Primenen. $17,1972,588-590)$.

[2] FuK, D.Kh., and S.V. NagaEv: Probability inequalities for sums of independent random variables. - Theory Probab. Appl. 16, 1971, 643-660 (English translation of Teor. Veroyatnost. i Primenen. 16, 1971, 660-675).

[3] Nagaev, S.V., and I.F. PInelis: Some inequalities for the distribution of sums of independent random variables. - Theory Probab. Appl. 22, 1977, 248-256 (English translation of Teor. Veroyatnost. i Primenen. 22, 1977, 254-263).

[4] Petrov, V.V.: Limit theorems for sums of independent random variables. - Nauka, Moscow, 1987 (Russian).

[5] Rosenthal, H.P.: On the subspaces of $L^{p}(p>2)$ spanned by sequences of independent random variables. - Israel J. Math. 8, 1970, 273-303.

[6] Rosenthal, H.P.: On the span in $L^{p}$ of sequences of independent random variables. Proceedings of the Sixth Berkeley Symposium on Mathematical Statistics and Probability, University of California Press, Berkeley-Los Angeles, 1972, Volume II, 149-167. 